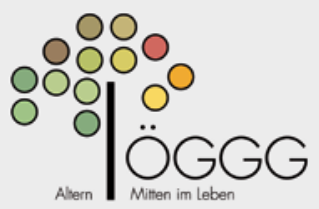

Z Gerontol Geriat 2012 • 45:574-574 DOI 10.1007/s00391-012-0370-Y

๑) Springer-Verlag 2012
Österreichische Gesellschaft für Geriatrie und Gerontologie (ÖGGG)

Mitglied der International Association of Gerontology and Geriatrics (IAGG)
Geschäftsstelle

Österreichische Gesellschaft

für Geriatrie und Gerontologie

Ilse Howanietz

Apollogasse 19

A-1070 Wien

ilse.howanietz@wienkav.at

www.geriatrie-online.at
Korrespondenzadresse

Prim. Dr. Katharina Pils

SMZ-Sophienspital

Apollogasse 19

A-1070 Wien

katharina.pils@wienkav.at

\title{
Tabuthema Gewalt
}

Das Tabuthema Gewalt an älteren Menschen in Einrichtungen des Sozial- und Gesundheitswesens wird in einer multiprofessionellen und interdisziplinären Arbeitsgruppe des Bundesministeriums für Arbeit, Soziales und Konsumentenschutz Österreich bearbeitet.

Die ÖGGG ist Mitherausgeber der Broschüre, die unter http://broschuerenservice.bmask. gv.at bestellt werden kann.

Die vorliegende Broschüre versucht sich dem Thema der Gewalt gegen ältere Menschen in Einrichtungen sorgsam zu nähern. Es geht nicht nur um klare Gewalthandlungen, sondern auch um den wachsamen Umgang mit älteren und betreuungsbedürftigen Personen. Das breite Spektrum reicht vom nachdrücklichen Anfassen, ungeduldigen
Eingeben von Speisen oder keine Unterstützung beim Essen gewähren bis hin zur fehlenden Privatsphäre oder einfach warten lassen. Es werden freiheitsbeschränkende Maßnahmen, monitäre Einschränkungen, Personalmangel, inadäquate Strukturen sowie traditionelle Wertevorstellungen diskutiert. Die vorliegende Publikation nimmt aber auch Bezug auf belastende Arbeitsbedingungen, strukturelle und persönliche Rahmenbedingungen, die Betreuungspersonen unterstützen und somit einen wertschätzenden Umgang mit komplexen PatientInnen erleichtern.

Eine Broschüre, die sensibilisiert ohne anzuklagen - ein wichtiger Beitrag um Gewaltbereitschaft frühzeitig zu erkennen.

\section{Neue Mitglieder}

20.-21. September 2011

Styria Geriatrica 2012

„Alter Mensch - neue Wege

in der Medizin"

Alte Universität Graz

http://www.medizin-medien.at/

dynasite.cfm?dsmid=104191

\section{5.-6. Oktober 2012}

11. Tagung für Allgemeinmedizin und Geriatrie

Börse, Wien

http://www.medizin-medien.at/ dynasite.cfm?dsmid=63816

19. Oktober 2012, 16.00 Uhr

8. Symposium der Altersmedizin „Bewegt im Alter“ LKH Gmunden

\section{Oktober 2012}

Fachtag Polypharmazie und Geriatrie

Schloss Schönbrunn

Tagungszentrum, Wien http://www.polypharmaziegeriatrie.at
25.-27. Oktober 2012

Geriatric Oncology:

Cancer in Senior Adults

12th Meeting of the International Society of Geriatric Oncology

Manchester, UK

www.siog.org

15. November 2012, 19.00 Uhr s.t. Wissenschaftlichen Sitzung der ÖGGG und des LBI für Altersforschung

„Ludwig Boltzmann-Institut für Altersforschung 1972-2012“

Gesellschaft der Ärzte,

Frankgasse 8, 1090 Wien

17. November 2012

Medicine of Ageing 2012

Stadtforum BTV, Gilmstrasse, Innsbruck

http://www.gerimed.at

21.-23. März 2013

Wiener Geriatriekongress

"Alter(n) - Chance und

Herausforderung"

Congress Center, Messe Wien

http://www.geriatriekongress.at

\author{
- Akad. GM DGKS Barbara Klammer \\ - Dr. Gerald Pichler \\ - Diätologin Elisabeth Proyer \\ - OA Dr. Ernst Ulsperger
}

